\title{
Aha! An Illuminating Perspective
}

\section{David Ginat (moderator) Tel-Aviv University ginat@post.tau.ac.il}

\author{
Dan Garcia \\ University of California at Berkeley \\ ddgarcia@cs.berkeley.edu
}

\author{
William Gasarch \\ University of Maryland \\ gasarch@cs.umd.edu
}

\section{Introduction}

The 'Aha!' phenomenon is familiar to us in many domains including computer science and mathematics (e.g., [2,3,6]). It often stems from an unexpected point of view that illuminates an appealing solution path. The 'Aha' reaction is common to all. Its occurrence is related to the problemsolvers' common perspectives and solution repertoires. Whether more frequent or less frequent, 'Aha' occurrences enrich and strengthen perspectives and repertoires in a stimulating manner.

Consider the following Ladder Problem: calculate the number of different ways to climb an N-stage ladder when each step is either one or two stages. One solution perspective may be 'forward reasoning', leading to a systematic accumulation of the possible climbing paths. Another perspective may be combinatorial, leading to the calculation of all the combinations of 1 and 2 that sum to $\mathrm{N}$. A third perspective may be 'backward reasoning', yielding recursive decomposition of the $\mathrm{N}^{\text {th }}$ case into the $\mathrm{N}-1$ and $\mathrm{N}$ 2 cases.

Some problem-solvers may fairly quickly invoke the third perspective and elegantly obtain the $\mathrm{N}^{\text {th }}$ Fibonacci number. Others may first follow one of the other perspectives and later realize the illuminating third perspective. The 'Aha' reactions among the solvers may vary. However, both less experienced and more experienced solvers will gain from recognizing the relevance and elegance of the recursive decomposition and enhance their problem-solving repertoires.

\section{Objective of the Special Session}

The goal of this special session is to emphasize and illustrate the importance of attempting various perspectives in algorithmic problem-solving. Novice problem-solvers tend to follow rather limited tracks in seeking a problem solution [7]. In algorithmic problem-solving such tracks often involve very common design techniques and design patterns [4]. While these techniques and patterns are essential, sole emphasis on their utilization may result in perceiving less common and less expected perspectives as magical 'Aha!' discoveries. We believe that such discoveries could be perceived differently, at least to some extent. A problem-solver that will realize a gradualadvancement process, which involves the seeking of diverse problem perspectives, will enrich her problem-solving repertoire and enhance her confidence in reaching illuminating discoveries.

\section{Nature of the Special Session}

The session will be an interactive seminar on illuminating problem-solving perspectives. The rationale and effectiveness of illuminating problem solving perspectives will be illustrated through colorful algorithmic challenges and their solutions. The session will include an introduction and interactive tutorials. The introduction will first display the 'Aha!' phenomenon and a short demonstration of different solution angles. The tutorials will involve presentations of algorithmic tasks that can be solved in different ways, encapsulating various simplicity and complexity (time and space) considerations. The audience will be encouraged to participate and offer solution perspectives. The arrival at illuminating insights will be achieved through gradual processes of various-angle examination, rather than via 'magical' discoveries.

One of the characteristics distinguishing between expert and novice problem-solvers is their ability (or inability) to thoroughly analyze a problem and view it from diverse perspectives. This is often referred to as lateral (vs. linear) thinking. A common means for developing novices' competence is apprenticeship. During apprenticeship one gradually acquires the values of the exercised solution process. The 'Aha' illuminations in the session will be reached in an apprenticeship manner, which can be applied in class.

The challenging tasks and their illuminating perspectives are particularly suited to CS1, CS2, Data Structures, and Algorithms courses. The colorful nature of the tasks makes them attractive pedagogically, and the illuminating characteristic of the perspectives classify them as essential tools for computer science educators. 


\section{Examples}

\section{Elections:}

The number of votes in the general elections in China is 100,000,000. The candidates are numbered from 1 to $1,000,000$. Given the sequence of the election votes, is there a candidate with a majority number of votes? You may read the input a couple of times.

This challenge is intriguing, as a simple solution based on keeping a huge array of counters (one for each candidate) is impossible. Several illuminating perspectives are considerably helpful. One is based on coarse-grain-finegrain counting; another - on binary decomposition, and a third - on inductive decomposition. (The thorough description appears in [5] and will therefore not be displayed in the special session.)

\section{Dinner:}

$\mathrm{N}$ people are invited to dinner and should be seated around a round table. Unfortunately, many of them are not in good terms with one another. Each person may have up to N/2-1 rivals. Find an arrangement to seat the people such that no one sits next to his rivals.

One way to solve this problem is by an exhaustive, backtracking search through all the possible seating arrangements. Another approach may involve reducing the problem into a graph problem and looking for a valid circular path. (Looks NP-Complete? It is not.) A third, very effective approach derives from a clever iteration based on an illuminating invariant property.

\section{Misére (reverse) Tic-Tac-Toe:}

This is an interesting twist on a children's paper and pencil game. Players take turns placing their respective $\mathrm{X}$ or $\mathrm{O}$ markers on an initially-empty $3 \times 3$ grid. The first to achieve three-in-a-row (vertically, horizontally or diagonally) loses. We are told that a win cannot be forced upon either the first or second player. That said, what is a good strategy for the first player to achieve the tie?

At first it seems that exhaustive search is the only means to determine the proper strategy. Most players agree that at the very least, the center should be avoided, as it has the most three-in-a-row axis lines through it. What makes this problem interesting is how wrong that intuition is; the loop invariant that always guarantees a tie is quite counterintuitive. The strategy has roots deep within economic \& combinatorial game theory and is called the "Tweedledee tweedledum" principle [1]. It is quite simple: the first player initially chooses the center square and then on every subsequent turn, picks the opposite (reflected about the center) free square.

\section{References}

1. Berlekamp, Elwyn; Conway, John; and Guy, Richard. Winning Ways for your Mathematical Plays, Academic Press, 1982.

2. Gardner, Martin, Aha! Insight, W. H. Freeman and Company, 1978.

3. Gardner, Martin, Aha! Gotcha, W. H. Freeman and Company, 1982.

4. Ginat, David, Early algorithm efficiency with design patterns, Computer Science Education, Vol. 11, No. 1, pp. 1-21, June 2001.

5. Ginat, David, On varying perspectives of problem decomposition, Proceedings of the $33^{\text {rd }}$ SIGCSE Technical Symposium on CS Education, February 2002.

6. Polya, George, How to Solve It, Princeton University Press, 1971 (re-issue).

7. Schoenfeld, Alan, Learning to think mathematically: problem solving metacognition, and sense making in mathematics, Handbook of Research on Mathematics Teaching and Learning (Macmillan), pp. 334-370, 1992. 